\title{
Cold-shock Protein Expression System Facilitates the Solubility of Human ST6Gal I in Escherichia Coli
}

\author{
Kei-ichi Watanabe, Kazuya I.P.J. Hidari* and Takashi Suzuki
}

Department of Biochemistry, University of Shizuoka, School of Pharmaceutical Sciences, and Global COE Program, 521 Yada, Suruga-ku, Shizuoka 422-8526, Japan

\begin{abstract}
The protein structures of most mammalian sialyltransferases have yet to be elucidated. Practical and convenient protein expression systems for soluble and active sialyltransferases will facilitate elucidation of the protein structures and catalytic mechanisms of these enzymes. The present study was performed to establish an efficient expression system for human ST6Gal I (hST6Gal I). cDNA encoding a soluble form of hST6Gal I was introduced into the bacterial expression vector pCold I carrying the cold shock promoter that is inducible by low-temperature conditions. The resultant DNA encodes the enzyme fused in frame with a maltose-binding protein (MBP) as a purification tag. This expression plasmid was introduced into the E. coli strain pGro7/BL21 harboring the molecular chaperones GroES and GroEL. Combined use of chaperone proteins and low-temperature cultivation during IPTG induction significantly improved the functional enzyme solubility in bacteria. The MBP-tagged hST6Gal I was efficiently purified by affinity chromatography using amyloseconjugated agarose.
\end{abstract}

Keywords: Sialyltransferase, cold shock vector, low-temperature induction, bacterial expression system.

\section{INTRODUCTION}

The sialyltransferase ST6Gal I catalyzes the transfer reaction of sialic acid to the non-reducing terminal Gal $\beta 1$ 4GlcNAc residues of glycoconjugates with $\alpha 2,6$ linkage [1]. Carbohydrate structures containing Neu5Ac $\alpha 2-6$ residues play critical roles in many biological events such as cell-cell recognition, cancer metastasis, immunological responses, and cell-pathogen interactions [2-9]. In addition, medical applications of sialyltransferase, such as modification of therapeutic proteins, have attracted a great deal of attention in recent years [10-12]. To understand the catalytic mechanisms of action of sialyltransferase, it is indispensable to elucidate the protein structures of these enzymes. Very recently, the protein structures of some bacterial sialyltransferases [13-15] and a mammalian $\alpha 2,3$ sialyltransferase [16] have been reported. However, the structures of other sialyltransferases, including human ST6Gal I (hST6Gal I), have not been determined. A convenient and efficient ST6Gal I expression system is indispensable to elucidate the protein structures and catalytic mechanisms of these enzymes.

In this study, we established an efficient expression and purification system for soluble and active recombinant hST6Gal I in Escherichia coli utilizing the bacterial expression vector pCold I. This vector significantly contributed to the production of soluble and active recombinant hST6Gal I.

*Address correspondence to this author at the Department of Biochemistry, School of Pharmaceutical Sciences, University of Shizuoka, 52-1 Yada, Suruga-ku, Shizuoka 422-8526, Japan; Tel: +81-54-264-5720;

Fax: +81-54-264-5723, E-mail: hidari@u-shizuoka-ken.ac.jp

\section{MATERIALS AND METHODS}

\section{Materials}

Pfu DNA polymerase was purchased from Promega (Madison, WI). Rat liver $\alpha 2,6$ sialyltransferase was purchased from Wako Pure Chemical Industries, Ltd. (Osaka, Japan). Rabbit anti-MBP antibody was purchased from New England Biolabs, Inc. (Ipswich, MA). Biotin-conjugated lectin, Sambucus sieboldiana agglutinin (SSA), was purchased from Seikagaku Corporation (Tokyo, Japan). All other chemicals were of the highest purity available.

\section{Cloning of the Gene Encoding MBP and Soluble form of ST6Gal I}

The primers for polymerase chain reaction (PCR) were designed according to the nucleotide sequence of hST6Gal I. The sequences of primers used to amplify the soluble form of hST6Gal I cDNA were as follows: forward primer containing a BamHI site (5'-TCATGGATCCGAATTCCAGGTGTTAAAGAG-3'), reverse primer containing an $\mathrm{XbaI}$ site (5'-TCATTCTAGATTAGCAGTGAATGGTCCGG-3').

PCR was performed using the cDNA in pSTMX [17] as a template for 25 cycles of denaturation $\left(94^{\circ} \mathrm{C}, 1 \mathrm{~min}\right)$, annealing $\left(51^{\circ} \mathrm{C}, 1 \mathrm{~min}\right)$, and extension $\left(72^{\circ} \mathrm{C}, 1.5 \mathrm{~min}\right)$, followed by incubation at $4^{\circ} \mathrm{C}$. The amplified DNA was ligated with linearized pGEM-T vector (Promega) according to the manufacturer's instructions. The nucleotide sequence of the isolated cDNA clone was confirmed by sequencing of both strands.

MBP was amplified by PCR using the following primers: forward primer containing an NdeI site (5'-TCATCATATG- 
AAAATAAAAACAGGTGC-3'), reverse primer containing a BamHI site and a cleavage site for HRV 3C protease (5'TCATGGATCCGGAGGGCCCCTGGAACAGAACTTCC AGCGCCGCCGCAGTCTGCGCGTCTTTCAG-3'). The amplified DNA fragment was ligated into the pGEM-T vector and confirmed by sequencing.

\section{Construction of Expression Vector}

The expression vector for recombinant hST6Gal I was prepared as follows. The DNA fragment encoding the soluble form of hST6Gal I was excised from hST6Gal I-pGEM$\mathrm{T}$ with BamHI/XbaI digestion, and purified by agarose gel electrophoresis. The DNA fragment encoding MBP was excised from MBP-pGEM-T with NdeI/BamHI digestion, and purified by agarose gel electrophoresis. The hST6Gal I fragment and MBP fragment were then subcloned into the NdeI - XbaI sites of the expression vector pCold I.

\section{Establishment of a Bacterial Clones Expressing hST6Gal I}

The BL21-derived E. coli strain pGro7/BL21 (Takara Bio, Inc., Otsu, Japan) expressing GroEL and GroES was used as the host. We introduced a plasmid harboring the gene encoding the soluble form of hST6Gal I and MBP. The resultant clone was designated hST6Gal I/pGro7. We also introduced the same plasmid into similar E. coli strains, including pTf16/BL21, pG-Tf2/BL21, pKJE7/BL21, and pGKJE8/BL21 and obtained hST6Gal I/pTf16, hST6Gal I/pGTf2, hST6Gal I/pKJE7, and hST6Gal I/pKJE8, respectively.

\section{Expression of Soluble hST6Gal I Protein}

In small-scale culture, a single colony of each clone was inoculated into Luria Broth (LB) medium supplemented with $100 \mu \mathrm{g} / \mathrm{mL}$ of ampicillin and $20 \mu \mathrm{g} / \mathrm{mL}$ chloramphenicol (LBAmpCm), and precultured at $37^{\circ} \mathrm{C}$ for overnight. Then, $0.5 \mathrm{~mL}$ of preculture was added to $9.5 \mathrm{~mL}$ of freshly prepared LBAmpCm supplemented with $0.5 \mathrm{mg} / \mathrm{mL}$ arabinose or $0.5 \mathrm{ng} / \mathrm{mL}$ tetracycline (according to the manufacturer's instructions), and cultured at $37^{\circ} \mathrm{C}$ until $\mathrm{OD}$ at $600 \mathrm{~nm}$ $\left(\mathrm{OD}_{600}\right)$ reached $0.4-0.5$. The protein expression in bacteria was induced at $15^{\circ} \mathrm{C}$ for $24 \mathrm{~h}$ in the presence of IPTG at a final concentration of $1 \mathrm{mM}$. In large-scale culture for purification of the enzyme, several colonies on the clone plate were inoculated into $50 \mathrm{~mL}$ of LBAmpCm and cultured overnight at $37^{\circ} \mathrm{C}$. Then, $25 \mathrm{~mL}$ of preculture was added to $475 \mathrm{~mL}$ of freshly prepared LBAmpCm supplemented with $0.5 \mathrm{mg} / \mathrm{mL}$ arabinose or $0.5 \mathrm{ng} / \mathrm{mL}$ tetracycline and cultured at $37^{\circ} \mathrm{C}$ until $\mathrm{OD}_{600}=0.4-0.5$. The protein expression in bacteria was induced at $15^{\circ} \mathrm{C}$ for $24 \mathrm{~h}$ in the presence of IPTG at a final concentration of $1 \mathrm{mM}$.

\section{ELISA-based Sialyltransferase Assay}

Quantitative enzyme assay for immobilized glycoproteins as acceptors was carried out as described previously [18, 19]. Desialylated $\alpha 1$-acid glycoprotein as an acceptor at a concentration of $10 \mu \mathrm{g} / \mathrm{mL}$ was immobilized on 96-well plastic plates (Maxisorp; Nunc Inc., Roskilde, Denmark). The enzyme reaction was carried out in the wells according to the procedure of Weinstein et al. [20] with slight modifications. The reaction mixture had the following final concentrations in a total volume of $50 \mu \mathrm{L}$ : $50 \mu \mathrm{M}$ CMP-Neu5Ac, $0.5 \%$ (w/v) Triton CF-54, 25 mM MES-NaOH, pH 6.5, and $0.6 \mu \mathrm{g}$ of target protein.

\section{Purification of Soluble Recombinant hST6Gal I}

All operations described below were carried out at $4{ }^{\circ} \mathrm{C}$ or on ice. For small-scale preparation of the soluble fraction, bacterial cell pellets were suspended in $10 \mathrm{~mL}$ of a sonication buffer containing $150 \mathrm{mM} \mathrm{NaCl}, 1 \mathrm{mM}$ benzamidine, 1 $\mathrm{mM}$ phenylmethylsulfonyl fluoride (PMSF), and $20 \mathrm{mM}$ Tris- $\mathrm{HCl}(\mathrm{pH} 7.5)$ and pulse-disrupted using an ultrasonifier until the solution became clear. The solution was centrifuged and the supernatant was used for the sialyltransferase assay.

For large-scale production, the bacterial cell pellet was suspended in $100 \mathrm{~mL}$ of sonication buffer and pulsedisrupted with an ultrasonifier until the solution become clear. The solution was centrifuged and the supernatant was subjected to affinity column chromatography as follows. The supernatant of induced $E$. coli was applied to a column of amylase-conjugated agarose equilibrated in a buffer (TBS) containing $150 \mathrm{mM} \mathrm{NaCl}, 25 \mathrm{mM}$ Tris- $\mathrm{HCl}(\mathrm{pH} 7.5)$. The retained sialyltransferase was eluted with TBS containing 10 $\mathrm{mM}$ maltose. Fractions containing hST6Gal I were collected and dialyzed against TBS. Then, the fraction was reloaded onto the amylase conjugated agarose in a similar manner. Finally, hST6Gal I was eluted, mixed to give a final concentration of $50 \%$ glycerol and $0.08 \%$ Triton CF-54, and stored at $-80^{\circ} \mathrm{C}$, until use.

\section{Digestion with HRV 3C Protease}

One unit of HRV 3C protease (Novagen, Darmstadt, Germany) was used for digestion of purified hST6Gal I. HRV 3C was added to purified hST6Gal I in HRV 3C protease cleavage buffer $(150 \mathrm{mM} \mathrm{NaCl}, 50 \mathrm{mM}$ Tris- $\mathrm{HCl}, \mathrm{pH}$ 7.5). The reaction mixture was incubated overnight at $4^{\circ} \mathrm{C}$. The reaction products were then analyzed by SDS-PAGE and Coomassie brilliant blue staining.

\section{RESULTS}

\section{Strategy for Cloning of the Gene Encoding Soluble hST6Gal I Into the Bacterial Vector pCold I}

The soluble form of the enzyme was fused to maltosebinding protein (MBP) as a purification tag. We introduced the genes encoding MBP and the soluble form of hST6Gal I into the plasmid expression vector pCold I (Fig. 1). The plasmid DNA was introduced into the BL21-derived E. coli strain pGro7/BL21, which expresses GroEL and GroES. The resultant host bacteria carry the plasmid harboring the gene encoding the soluble form of hST6Gal I and MBP, and the obtained clone was designated hST6Gal I/pGro7. We also introduced the same plasmid DNA into other E. coli strains, i.e., pTf16/BL21, pG-Tf2/BL21, pKJE7/BL21, pGKJE8/BL21, resulting in hST6Gal I/pTf16, hST6Gal I/pGTf2, hST6Gal I/pKJE7, and hST6Gal I/pKJE8.

\section{Expression of the Soluble form of MBP-tagged hST6Gal I}

When BL21 was used as the bacterial host, MBP-tagged hST6Gal I was not expressed in the soluble fraction (data not shown). Therefore, we attempted to express MBP-tagged hST6Gal I in the soluble fraction using the chaperone coex- 


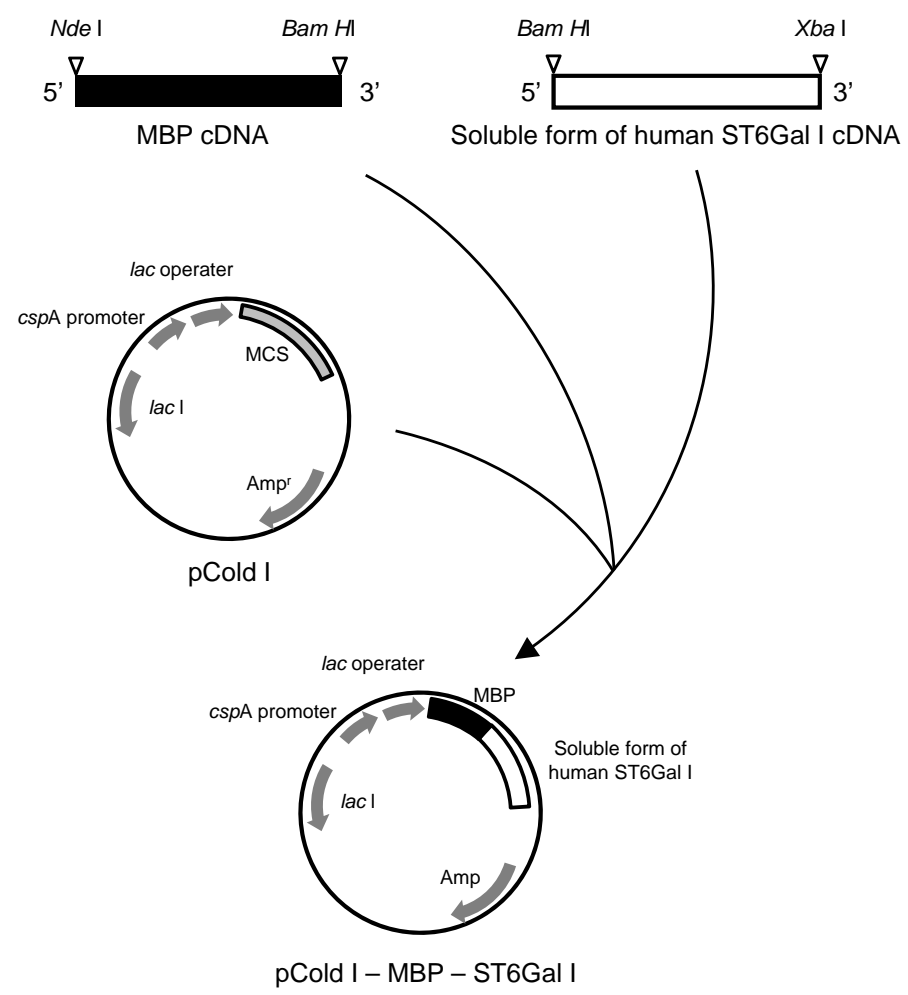

Fig. (1). Plasmid map of the expression vector. The genes coding MBP and hST6Gal I were inserted into the cloning site of the bacterial expression vector pCold I.

pression system. After protein induction in the above bacterial strains at $15^{\circ} \mathrm{C}$, bacteria were sedimented and the pellets were disrupted by ultrasonication. Expression of MBPtagged hST6Gal I in each bacterial host was analyzed by western blotting using anti-MBP antibody (Fig. 2). MBPtagged hST6Gal I was expressed at high levels in hST6Gal I/pGro7 (lane 1) and hST6Gal I/pG-KJE8 (lane 4), and lowlevel expression was observed in hST6Gal I/ pKJE7 (lane 3). On the other hand, no expression was detected in either hST6Gal I/pG-Tf2 (lane 2) or hST6Gal I/pTf16 (lane 5). Sialyltransferase activities in the supernatants were determined by ELISA-based assay as described previously [18, 19]. As shown in Fig. (3), the supernatant of hST6Gal I/pGro7 showed the highest levels of activity $(55.7 \mu \mathrm{U} / \mathrm{mg}$ protein/min). hST6Gal I/pG-KJE8 and hST6Gal I/pKJE7 showed lower levels of enzyme activity. These data were in good agreement with those obtained by western blotting analysis (Fig. 2). These observations indicated that hST6Gal I/pGro7 was the most effective host for expression of the MBP-tagged hST6Gal I. Although hST6Gal I/pG-Tf2 or hST6Gal I/pTf16 had slightly enzyme activity, the signals corresponded to the enzyme were not detected on a membrane with anti-MBP antibody. It was because the enzyme protein expression in those host bacteria may be less than the detection level by immunoblotting anaysis.

\section{Purification of a Soluble form of MBP-tagged hST6Gal I from Bacteria}

hST6Gal I/pGro7 pellets prepared from $500 \mathrm{~mL}$ of culture were disrupted with ultrasonication. The supernatant thus obtained was subjected to amylose-conjugated agarose column chromatography. MBP-tagged hST6Gal I was par- tially purified by affinity chromatography (Fig. 4, lane 1). Western blotting analysis of the purified fraction with antiMBP antibody showed that the maltose-eluted fraction contained MBP-tagged ST6Gal I (Fig. 4 lane 2). In this step, the tagged enzyme was obtained in a yield of $41.1 \%$ and with

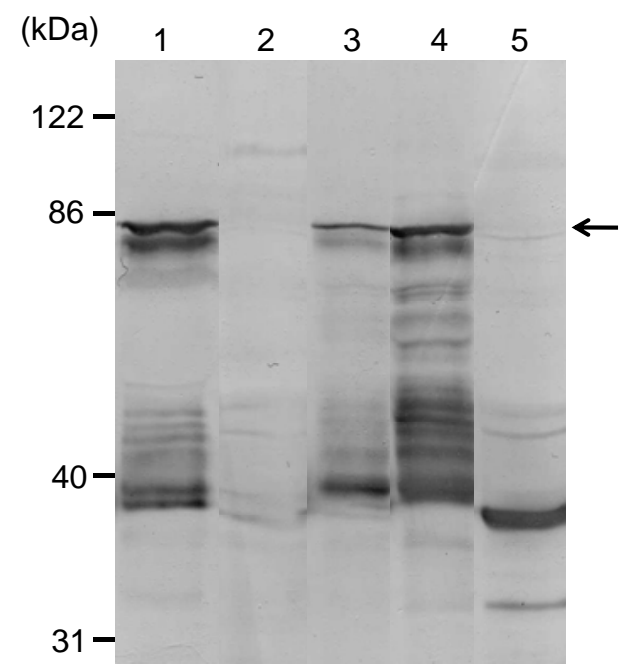

Fig. (2). ST6Gal I protein expression in chaperone-coexpressing $E$. coli. The protein expression in bacteria was induced at $15^{\circ} \mathrm{C}$ for 24 $\mathrm{h}$ in the presence of IPTG at a final concentration of $1 \mathrm{mM}$. Induced bacterial cells were disrupted by ultrasonication. Crude lysates cleared by sedimentation were collected and subjected to SDSPAGE, followed by western blotting analysis using anti-MBP antibody. Lane 1, pGro7/BL21; lane 2, pG-Tf2/BL21; lane 3, pKJE7/BL2; lane 4, pG-KJE8/BL21; lane5, pTf16/BL21. The arrow indicates MBP-tagged ST6Gal I. 


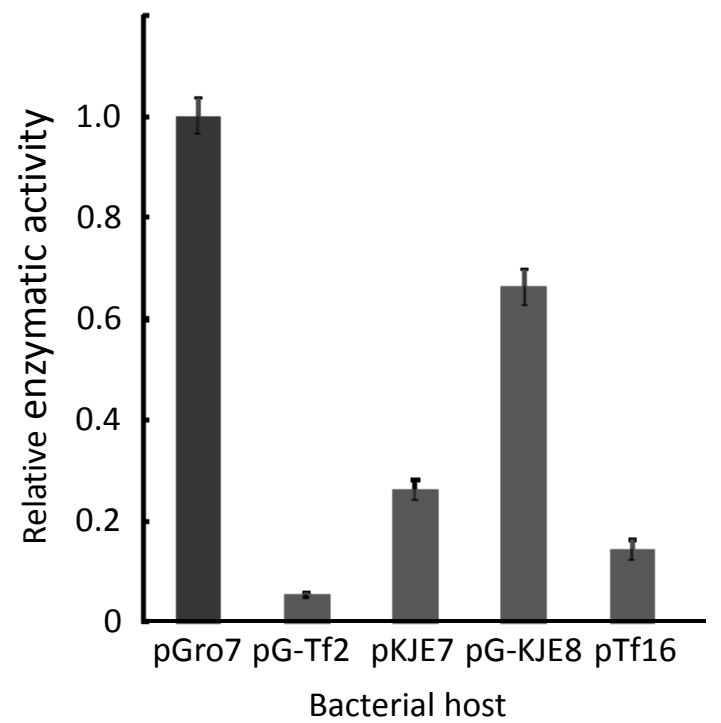

Fig. (3). Relative enzymatic activity of crude lysates prepared from host bacterial cells. The quantitative enzyme assay was carried out as described in Materials and methods. Activity of the host is shown relative to pGro7/BL21 as $1.0(55.7 \mu \mathrm{U} / \mathrm{mg} \mathrm{protein} / \mathrm{min})$.

A

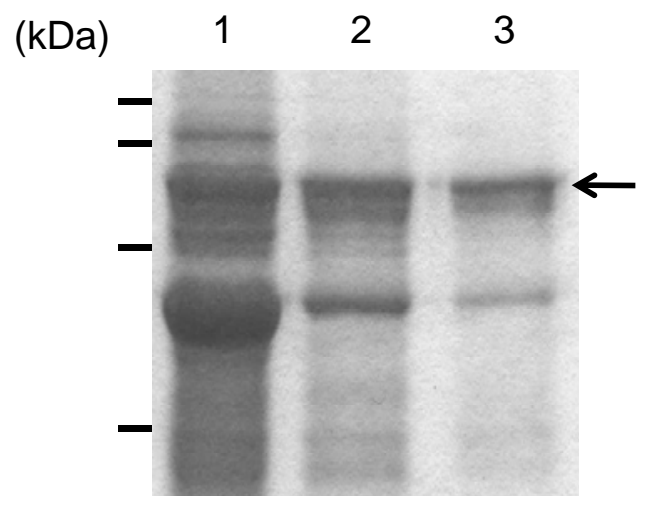

\section{Coomassie Brilliant Blue staining}

B

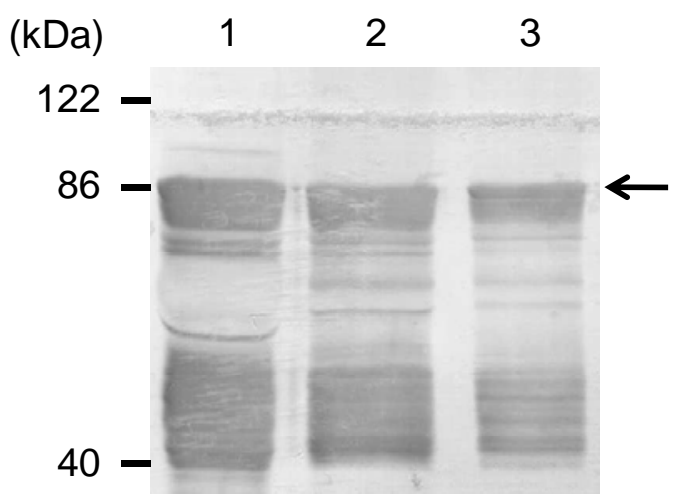

Western blotting

Fig. (4). Purification of MBP-tagged hST6Gal I by affinity chromatography using amylose-conjugated agarose. Crude lysate (lane 1) was loaded onto an amylose-conjugated agarose column. MBP-tagged ST6Gal I was eluted with TBS containing $10 \mathrm{mM}$ maltose. Elution (lane 2) was dialyzed against TBS to remove maltose. The obtained fraction was reloaded onto the amylose-conjugated agarose column. MBP-tagged ST6Gal I was eluted with TBS containing $10 \mathrm{mM}$ maltose (lane 3). Fractions were analyzed by SDS-PAGE (A) and Western blotting using anti-MBP antibody (B). Arrows indicate MBP-tagged ST6Gal I.

3.88-fold purification. The eluted fraction was dialyzed against MBS (150 mM NaCl, $20 \mathrm{mM}$ MES-NaOH, pH 6.0). The dialyzed fraction was again subjected to amyloseconjugated agarose column chromatography. Western blotting analysis with anti-MBP antibody demonstrated that the major fraction obtained by maltose elution. contained the MBP-tagged ST6Gal I (Fig. 4 lane 3). In this final purification step, the MBP-tagged ST6Gal I was obtained in a yield of $20.3 \%$ and with 6.12 -fold purification. The purification of MBP-tagged ST6Gal I is summarized in Table 1. The specific activity of the recombinant enzyme was $0.3 \mathrm{mU} / \mathrm{mg}$ protein $/ \mathrm{min}$. The overall yield of the enzyme was $1.13 \mathrm{mU}$ from $500 \mathrm{~mL}$ of culture medium. The specific activity was lower than that described in our previous study [17]. This is the reason why purification level of the enzyme was relatively lower.

\section{DISCUSSION}

There have been no previous reports of functional and soluble expression of mammalian glycosyltransferases, including sialyltransferases, in E. coli. When expressed in $E$. coli, the recombinant enzyme was transported into inclusion bodies, making it resistant to solubilization with mild detergents [21]. We established a soluble expression system for human sialyltransferase ST6Gal I in E. coli [17]. However, the yield of the recombinant protein was still low in this system. As a result, a large culture volume was required to obtain the enzyme in sufficient amounts for in vitro applica- 
Table 1. Summary of Purification of a MBP-tagged hST6Gal I

\begin{tabular}{|c|c|c|c|c|c|c|}
\hline Step & Volume (ml) & Total Protein (mg) & $\begin{array}{c}\text { Specific Activitya } \\
\text { ( } \mu \text { units/mg protein/min) }\end{array}$ & $\begin{array}{c}\text { Total Activity }^{\mathrm{a}} \\
\text { ( } \mu \text { units/min) }\end{array}$ & Yield (\%) & Fold \\
\hline Crude lysate & 100 & 100.6 & 55.7 & 5.57 & 100 & 1 \\
\hline $\begin{array}{l}\text { Partially purified enzyme } \\
\text { (first elution) }\end{array}$ & 55 & 10.58 & 216 & 2.29 & 41.1 & 3.88 \\
\hline $\begin{array}{l}\text { Partially purified enzyme } \\
\text { (second elution) }\end{array}$ & 35 & 3.31 & 341 & 1.13 & 20.3 & 6.12 \\
\hline
\end{tabular}

${ }^{a}$ One unit of activity is defined as $1 \mu \mathrm{mol}$ of sialic acid transfer to acceptor substrate per $1 \mathrm{~min}$ at $37^{\circ} \mathrm{C}$.

tions. The present study was performed to improve the expression of the soluble form of recombinant human ST6Gal I in bacteria. For this purpose, we used the cold shock plasmid vector pCold I, which has high efficiency of protein expression at temperatures below $15^{\circ} \mathrm{C}$. Our previous study demonstrated that induction below $25^{\circ} \mathrm{C}$ contributes to expression of soluble hST6Gal I in E. coli and prevents its digestion by proteases [17]. Therefore, protein expression at low temperatures with cold shock vector is advantageous for expression of soluble hST6Gal I. Some mammalian proteins such as human $\beta$-actin have been expressed using the cold shock vector [22]. Our results showed that combined use of cold shock vector and chaperone-coexpressing E. coli strains significantly improved solubilization of the recombinant protein. The chaperone-coexpressing E. coli strain pGro7/BL21 was the best host for expression of hST6Gal I using pCold I vector (Figs. 2 and 3).

The expressed protein was effectively purified by amylose-conjugated agarose column chromatography. The protein purified by amylose-conjugated column chromatography contained a few minor components with faster mobility on the gel detected with anti-MBP antibody, indicating that they

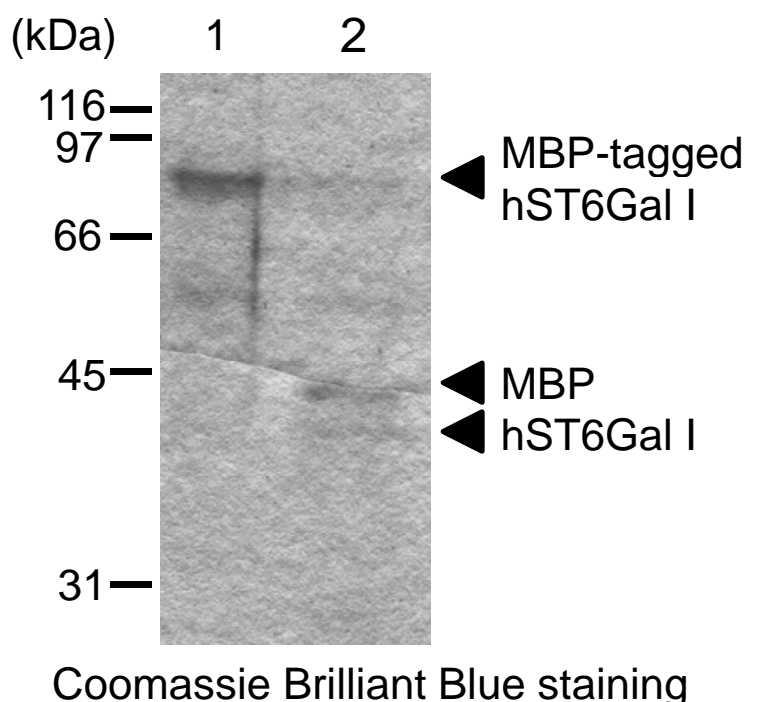

Fig. (5). Purified MBP-tagged hST6Gal I were treated with HRV 3C protease. Purified MBP-ST6Gal I were mixed with HRV3C protease (1unit/20 mg the protein of interset) and incubated overnight at $4^{\circ} \mathrm{C}$. Lane 1, purified MBP-tagged hST6Gal I; lane 2, purified MBP-tagged hST6Gal I treated with HRV 3C protease. Samples were analyzed by SDS-PAGE, followed by staing of the gel with coomassie brilliant blue reagent. were originally derived from MBP-tagged ST6Gal I and proteolytically degraded during purification. For further purification, HRV 3C protease could be useful as hST6Gal I does not contain sequences for recognition by this protease. A single cleavage site was introduced into the junction of MBP and hST6Gal I. We found that MBP-tagged hST6Gal I was digested to MBP and hST6Gal I by HRV 3C protease (Fig. 5). The combination of HRV 3C digestion and other types of affinity chromatography could provide highly purified enzyme in sufficient amounts for crystallization experiments.

In conclusion, an efficient bacterial expression system for soluble hST6Gal I was established. The recombinant enzymes could be used to study the molecular mechanisms of the catalytic reaction.

\section{ACKNOWLEDGMENTS}

This work was supported by grants-in-aid for Scientific Research on Priority Areas (21570146 to K.I.P.J.H.) from the Ministry of Education, Science, Sports, and Culture of Japan. This work was also supported by a Cooperative Research Grant (2010-A-7) from the Institute of Tropical Medicine, Nagasaki University.

\section{CONFLICT OF INTEREST}

None.

\section{REFERENCES}

[1] Harduin LA, Vallejo RV, Ange MKR, et al. The human sialyltransferase family. Biochimie 2001; 83: 727-37.

[2] Varki A. Biological roles of oligosaccharides: All of the theories are correct. Glycobiology 1993; 3: 97-130.

[3] Gagneux P, Varki A. Evolutionary considerations in relating oligosaccharide diversity to biological function. Glycobiology 1999; 9: 747-55.

[4] Dall'Olio F, Chiricolo M. Sialyltransferases in cancer. Glycoconjug J 2001; 18: 841-50.

[5] Varki NM, Varki A. Diversity in cell surface sialic acid presentations: implications for biology and disease. Laboratory Investig 2007; 87: 851-7.

[6] Suzuki Y. Gangliosides as influenza virus receptors. Variation of influenza viruses and their recognition of the receptor sialo-sugar chains. Prog Lipid Res 1994; 33: 429-57.

[7] Sperandio M, Frommhold D, Babushkina I, et al. $\alpha 2,3$ Sialyltransferase-IV is essential for L-selectin ligand function in inflammation. Eur J Immunol 2006; 36: 3207-15.

[8] Marathe DD, Chandrasekaran EV, La JTY, et al. System-level studies of glycosyltransferase gene expression and enzyme activity that are associated with the selectin binding function of human leukocytes. FASEB J 2008; 22: 1-14. 
[9] Hedlund M, Ng E, Varki A, Varki NM. $\alpha 2-6-$ linked Sialic Acids on $\mathrm{N}$-glycans Modulate Carcinoma Differentiation In vivo. Cancer Res 2008; 68: 388-94

[10] Elliott S, Lorenzin, T, Asher S, et al. Enhancement of therapeutic protein in vivo activities through glycoengineering. Nat Biotechnol 2003; $21: 414-21$

[11] Sato M, Furuike T, Sadamoto R, et al. Glycoinsulins: dendritic sialyloligosaccharide-displaying insulins showing a prolonged blood-sugar-lowering activity. J Am Chem Soc 2004; 126: 1401322.

[12] Lindhout T, Iqbal U, Willis LM, et al. Site-specific enzymatic polysialylation of therapeutic proteins using bacterial enzymes. Proc Natl Acad Sci USA 2011; 108: 7397-402.

[13] Chiu CP, Watts AG, Lairson LL, et al. Structural analysis of the sialyltransferase CstII from Campylobacter jujeni in complex with a substrate analog. Nat Strust Mol Biol 2004; 14: 163-70.

[14] Chiu CP, Lairson LL, Gilbert M, et al. Structural analysis of the alpha-2,3-sialyltransferase Cst-I from Campylobacter jujeni in apo and substrate-analogue bound forms. Biochemistry 2007; 46: 7196204.

[15] Kim DU, Yoo JH, Lee YJ, et al. Structural analysis of sialyltransferase PM0188 from Pasteurella multocida complexed with donor analogue and acceptor sugar. BMB Rep 2008; 41: 4854.
[16] Rao FV, Rich JR, Rakic B, et al. Structural insight into mammalian sialyltransferases. Nat Struct Mol Biol 2009; 16: 1186-8.

[17] Hidari KI, Horie N, Murata $\mathrm{T}$, et al. Purification and characterization of a soluble recombinant human ST6Gal I functionally expressed in Eschericha coli. Glycoconj J 2005; 22: 111.

[18] Mattox S, Walrath K, Ceiler D, et al. A solid-phase assay for the activity of CMP-NeuAc: Gal beta 1-4GlcNacR alpha-2,6sialyltransferase. Anal Biochem 1992; 206: 430-6

[19] Hidari KI, Oyama K, Ito $\mathrm{G}$, et al. Identification and characterization of flavonoids as sialyltransferase inhibitors. Biochem Biophys Res Commun 2009; 382: 609-13.

[20] Weinstein J, de Souza-e-Silva U, Paulson JC. Purification of a $\mathrm{Gal} \beta 1 \rightarrow 4 \mathrm{GlcNAc} \quad \alpha 2 \rightarrow 6 \quad$ sialyltransferase and a Gal $1 \rightarrow 3(4)$ GlcNAc $\alpha 2 \rightarrow 3$ sialyltransferase to homogeneity from rat liver. J Biol Chem 1982; 257: 13835-44.

[21] Hamamoto T, Lee Y-C, Kurosawa N, et al. Expression of mouse Gal beta 1,4GlcNAc alpha 2,6-sialyltransferase in an insoluble form in Escherichia coli and parcial renaturation. Bioorg Med Chem 1994; 2: 79-84.

[22] Tamura M, Ito K, Kunihiro S, et al. Production of human $\beta$-actin and a mutant using a bacterial expression system with a cold shock vector. Protein Exp Purif 2011; 78: 1-5.

(C) Watanabe et al.; Licensee Bentham Open.

This is an open access article licensed under the terms of the Creative Commons Attribution Non-Commercial License (http://creativecommons.org/licenses/by-nc/3.0/) which permits unrestricted, non-commercial use, distribution and reproduction in any medium, provided the work is properly cited. 